\title{
A massless neutrino and lepton mixing patterns from finite discrete subgroups of $\mathrm{U}(3)$
}

\author{
Anjan S. Joshipura ${ }^{a}$ and Ketan M. Patel ${ }^{b}$ \\ ${ }^{a}$ Physical Research Laboratory, Navarangpura, \\ Ahmedabad 380 009, India \\ ${ }^{b}$ Istituto Nazionale Fisica Nucleare, Sezione di Padova, \\ I-35131 Padova, Italy \\ E-mail: anjan@prl.res.in, ketan.patel@pd.infn.it
}

Abstract: Finite discrete subgroups of $\mathrm{U}(3)$ as possible flavour symmetries $G_{f}$ for a massless neutrino with predictive mixing angles are studied. This is done by assuming that a residual symmetry $S_{\nu}$ appropriate for describing a massless neutrino is contained in $G_{f}$. It is shown that all the groups $G_{f}$ admitting three dimensional faithful irreducible representation and generated from a specific set of $3 \times 3$ matrices imply only one of the three flavour compositions for the massless state namely, unmixed, maximally mixed with equal probabilities and bimaximally mixed with probabilities $(0,1 / 2,1 / 2)$ and their permutations. This result holds irrespective of the order of $G_{f}$ and the choice of $S_{\nu}$ within it. All of these lead to unfavorable leading order prediction for the solar mixing angle. Neutrino mixing pattern is then numerically investigated in case of subgroups of $U(3)$ with order less than 512 and it is found that only one of these can lead to a massless neutrino and leading order predictions for all the mixing angles close to their experimental values. Ways to correct for the solar angle prediction are proposed and two concrete examples giving the observed mixing pattern are discussed.

Keywords: Neutrino Physics, Discrete and Finite Symmetries

ARXIV EPRINT: 1401.6397 


\section{Contents}

1 Introduction 1

2 Massless neutrino and flavour symmetries 3

3 Massless neutrino and lepton mixing from discrete subgroups of U(3) 4

3.1 Massless state: general flavour structure and implications 4

$\begin{array}{lll}3.2 & \text { Mixing patterns with a massless neutrino } & 7\end{array}$

4 A numerical scan of discrete subgroups of $\mathrm{U}(3) \quad 10$

$\begin{array}{llr}4.1 & \text { Results of the groups in category (X) } & 10\end{array}$

$\begin{array}{lll}4.2 & \text { Results of the groups in category (Y) } & 12\end{array}$

5 Modifying solar mixing angle: two alternatives $\quad \mathbf{1 3}$

$\begin{array}{lll}5.1 & \text { Discrete subgroups of } \mathrm{U}(2) \times \mathrm{U}(1) & 13\end{array}$

$\begin{array}{ll}5.2 \text { Modified neutrino mass matrices } & 15\end{array}$

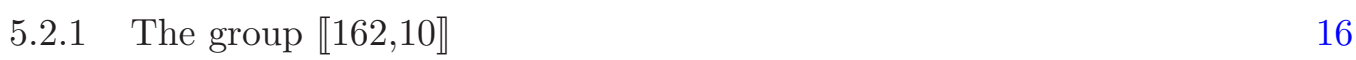

$\begin{array}{lll}5.2 .2 & \text { The group } S_{4}(2) & 17\end{array}$

$\begin{array}{lll}6 & \text { Summary } & 19\end{array}$

\section{Introduction}

The orderly pattern found in leptonic mixing may be signaling the existence of some underlying flavour symmetry, see [1-5] for some recent reviews. The mixing pattern is quite well-known by now but it is still difficult to fix a discrete symmetry responsible for it. A systematic approach to look for such symmetries has been pursued vigorously [6-16]. Basic idea in this approach is to (a) first obtain residual symmetries $G_{\nu}$ and $G_{l}$ of the neutrino and the charged lepton mass matrices based on the observed mixing pattern and (b) look for the flavour symmetry group $G_{f}$ which contain $G_{\nu}, G_{l}$ as subgroups. $G_{\nu}$ is usually a $Z_{2} \times Z_{2}$ symmetry in case of three massive Majorana neutrinos with unconstrained masses and $G_{l}$ is some discrete subgroup of $\mathrm{U}(1) \times \mathrm{U}(1) \times \mathrm{U}(1)$. The advantage of this approach is that mixing pattern is completely fixed by the choice of $G_{\nu}$ and $G_{l}$ without any detailed knowledge of the underlying theory. Moreover, a general prescription can be formulated [17] which leads to the desired mixing from some underlying Lagrangian invariant under $G_{f}$ spontaneously broken to $G_{\nu}$ and $G_{l}$. This basic framework has been used [1-16] to obtain zeroth order mixing patterns such as tri-bimaximal (TBM) and bimaximal (BM) and also to obtain some reasonable predictions on the mixing angles but flavour groups which can 
predict all the mixing angles in leading order within their $2 \sigma$ values are very few and large with order $>600[18]$.

The above framework does not put any restrictions on neutrino masses. The present neutrino data are still consistent with the quasi degenerate neutrinos or a spectrum with a massless neutrino both in case of the normal and inverted mass hierarchies. It would be appropriate to look for the modification of the above scenario which yields either of these spectrum. The case of the quasi degenerate neutrino was considered in [19] while the flavour symmetries appropriate for obtaining a massless state were discussed in [20]. Both, the choices for the residual symmetries and the flavour groups become different in theses cases. If neutrinos are Majorana particles and one of them is massless then one can redefine the phase of the massless field. Thus, one of the $Z_{2}$ symmetries of the neutrino mass matrix used as a member of $G_{\nu}$ in the usual approach gets replaced by a $Z_{n}$ symmetry with the corresponding $3 \times 3$ matrix $S_{\nu}$ having Det. $\left(S_{\nu}\right) \neq \pm 1$. As a result, the group embedding this also needs to be a discrete subgroup (DSG) of $\mathrm{U}(3)$ rather than that of $\mathrm{SU}(3)$. This idea was elaborated in [20] and the group series $\Sigma\left(2 n^{2}\right)$ [5] and $\Sigma\left(3 n^{3}\right)$ as well as the group $S_{4}(2)$ [21] were studied as possible flavour symmetries of the massless neutrino. The groups $\Sigma\left(2 n^{2}\right)$ were shown to lead to a neutrino mass matrix with $\mu-\tau$ symmetry for arbitrary $n$. The groups $\Sigma\left(3 n^{3}\right)$ were shown to lead to democratic mixing while $S_{4}(2)$ provided an example of symmetry leading to the BM mixing pattern. None of these groups could predict all three mixing angles correctly but both $S_{4}(2)$ and $\Sigma\left(2 n^{2}\right)$ could yield a good zeroth order predictions and perturbations leading to the correct mixing patterns were also studied in case of $S_{4}(2)$.

Aim of the present paper is to make an exhaustive search for an appropriate flavour group and see if a massless neutrino and realistic mixing angles can be obtained with some flavour symmetry. This search leads to a "no-go result" which demonstrates that the solar angle cannot be predicted correctly at the leading order in a very large class of groups to be specified as we go along. In all these groups, the flavour composition of the massless state gets determined irrespective of the nature of the group and for all possible choices of $G_{\nu}$ and $G_{l}$ within it. The allowed compositions are only three types. The trimaximal with probability to be in different flavour states as $\frac{1}{3}(1,1,1)$ or bimaximal with probabilities $\frac{1}{2}(0,1,1)$ and its permutations and trivial one $(1,0,0)$ and its permutations. This immediately results in wrong prediction for the solar angle in case of the normal hierarchy. In the case of inverted hierarchy, bimaximally mixed massless state provides a good leading order approximations to the reactor and atmospheric mixing angles but the solar angle turns out to be maximal as we will show later. This feature follows in a large class of groups which are DSG of U(3) having a three dimensional faithful irreducible representation (IR). In particular, this result holds for all but one group having order $<512$.

This paper is organized as the follows. We briefly review the idea of predicting a massless neutrino within the realm of the discrete symmetries in the next section. In section 3, we provide a simple analytic study of the large class of DSG of U(3) and discuss 
the allowed mixing patterns with a massless neutrino. The predictions of lepton mixing angles obtained by an exhaustive numerical scan of small DSG of U(3) are presented in section 4 . We discuss in section 5 some alternatives which can lead to correct solar mixing angles as well and discuss specific scenario capable of reproducing all three mixing angles in the experimentally allowed range. Finally, we summarize our study in section 6 .

\section{Massless neutrino and flavour symmetries}

We first review a framework relating flavour symmetries to neutrino mixing and its generalization which can also yield a massless neutrino. Assume that the neutrino (charged lepton) mass matrix in arbitrary basis is invariant under a set of unitary generators $S_{i}\left(T_{\alpha}\right)$ which form a discrete group $G_{\nu}\left(G_{l}\right)$. All $S_{i}$ are assumed to commute and are diagonalized by a matrix $V_{\nu}$. Similarly, the commuting set $T_{\alpha}$ is diagonalized by $V_{l}$. The invariance of the leptonic mass matrices under the respective groups can be used to show that the neutrino mixing matrix $U_{\text {PMNS }}$ is determined in terms of $V_{\nu}$ and $V_{l}$ [6-16]:

$$
U_{\mathrm{PMNS}}=P_{l} V_{l}^{\dagger} V_{\nu} P_{\nu}
$$

where $P_{l}$ and $P_{\nu}$ are arbitrary diagonal phase matrices.

The minimal group $G_{\nu}$ can be constructed from the observation that mass terms for the Majorana neutrinos remain unchanged under a change of sign of any of the neutrino mass eigenstates. Thus neutrino mass matrix in the mass basis is trivially invariant under

$$
s_{1}=\text { Diag. }(1,-1,-1), \quad s_{2}=\text { Diag. }(-1,1,-1) \text { and } s_{3}=s_{1} s_{2},
$$

where $\operatorname{Det}\left(s_{i}\right)$ is chosen +1 . Any two of these define a $Z_{2} \times Z_{2}$ symmetry. This implies that

$$
S_{i}=V_{\nu} s_{i} V_{\nu}^{\dagger}
$$

correspond to $G_{\nu}=Z_{2} \times Z_{2}$ symmetry in a basis labeled by $V_{\nu}$. This along with the

$$
T_{l}=V_{l} \text { Diag. }\left(e^{i \phi_{e}}, e^{i \phi_{\mu}}, e^{i \phi_{\tau}}\right) V_{l}^{\dagger},
$$

with $\phi_{e, \mu, \tau}$ being some discrete phases can be used as residual symmetries to be embedded in a bigger group $G_{f}$.

When one of the neutrinos is massless, then phase of the corresponding field can be changed without affecting neutrino mass term. In this case, one of the $S_{i}$ say $S_{1}$ can be replaced by $[20]$

$$
S_{1 \nu}=V_{\nu} \operatorname{Diag} \cdot(\eta, 1,-1) V_{\nu}^{\dagger},
$$

with $\eta^{N}=1$ and $N \geq 3$. The $S_{1 \nu}$ forms a $Z_{N}\left(Z_{2 N}\right)$ group for even (odd) $N$. This along with $S_{2,3}$ provide possible residual symmetries if one neutrino is massless. We are assuming here that the two massive neutrinos are non-degenerate. $S_{\nu}$ has to be chosen differently if this not the case [19]. We shall not entertain this possibility here. 
Note that, all the eigenvalues of $S_{1 \nu}$ as defined above are different. This uniquely fixes all the mixing angles in $V_{\nu}$ and it is sufficient to take this as a residual symmetry of the neutrino mass matrix and look for embedding of this $G_{\nu}$ and $G_{l}$ into a bigger group $G_{f}$. Arbitrariness in one of the mixing angles would remain if $S_{1 \nu}$ above is replaced by

$$
S_{1 \nu}^{\prime}=V_{\nu} \operatorname{Diag} .(\eta, 1,1) V_{\nu}^{\dagger} .
$$

We shall use either $S_{1 \nu}$ or $S_{1 \nu}^{\prime}$ as possible $Z_{N}$ in the following. As for $G_{l}$, we shall choose $T_{l}$ defined in eq. (2.4) which uniquely fixes $V_{l}$ if all phases are different. We then look for the discrete subgroups of U(3) which contain $T_{l}$ and $S_{1 \nu}$ or $S_{1 \nu}^{\prime}$ as elements. A complete classification of all discrete subgroups of $\mathrm{U}(3)$ is not available but our analysis would encompass all the known DSG of U(3) with a three dimensional faithful irreducible representation and many having (2+1)-dimensional reducible representation [21, 22].

\section{Massless neutrino and lepton mixing from discrete subgroups of $U(3)$}

Let us first review here some available information on the DSG of U(3) which will be used in our analysis. Details can be found in [23, 24] and specially in [21]. All the DSG of U(3) which are also the subgroups of $\mathrm{SU}(3)$ have been classified. The complete list can be found for example in [23-27]. They are characterized in terms of some small set of $3 \times 3$ matrices which are used to generate elements of various DSG of SU(3). The present knowledge on DSG of $\mathrm{U}(3)$ which are not the subgroups of $\mathrm{SU}(3)$ is partial. We will concentrate in this section on such subgroups of $\mathrm{U}(3)$ admitting a faithful three dimensional irreducible representation. It is convenient to divide these groups in two categories:

(X) Those which are generated by a specific set of generators $R, S, T, U, V$ and $W$ as given in [21] and reproduced here in table 1 and

(Y) those which are generated by more special textures labeled as $X_{1}, \ldots, X_{10}$ in table 1.

All the groups which can be generated using set (X) are not known but the groups with order $<512$ generated by the set $(\mathrm{X})$ and $(\mathrm{Y})$ and which cannot be written as direct product of $F \times Z_{n}$ are listed by Ludl [21]. One can obtain groups $F \times Z_{n}$ from this set as discussed by Ludl. There are 75 groups with order $<512$ of which only 5 fall in the category (Y). In addition to these 75 groups, Ludl has also identified [21] infinite series of groups called $S_{4}(n), T_{m}(n), \Delta(n, j), \Delta(n, j, k)$ which are expressible as semi-direct product of DSG of SU(3) with some cyclic group $Z_{m}$. All these series fall in the category (X).

\subsection{Massless state: general flavour structure and implications}

We now state and derive a general theorem regarding the structure of the massless state that holds for a large class of DSG of U(3).

Theorem. If $S_{1 \nu}$ and $T_{l}$ are elements of any group $G_{f}$ in category $(X)$ generated by combination of matrices $R, S, T, U, V, W$ listed in table 1 and if all the eigenvalues 


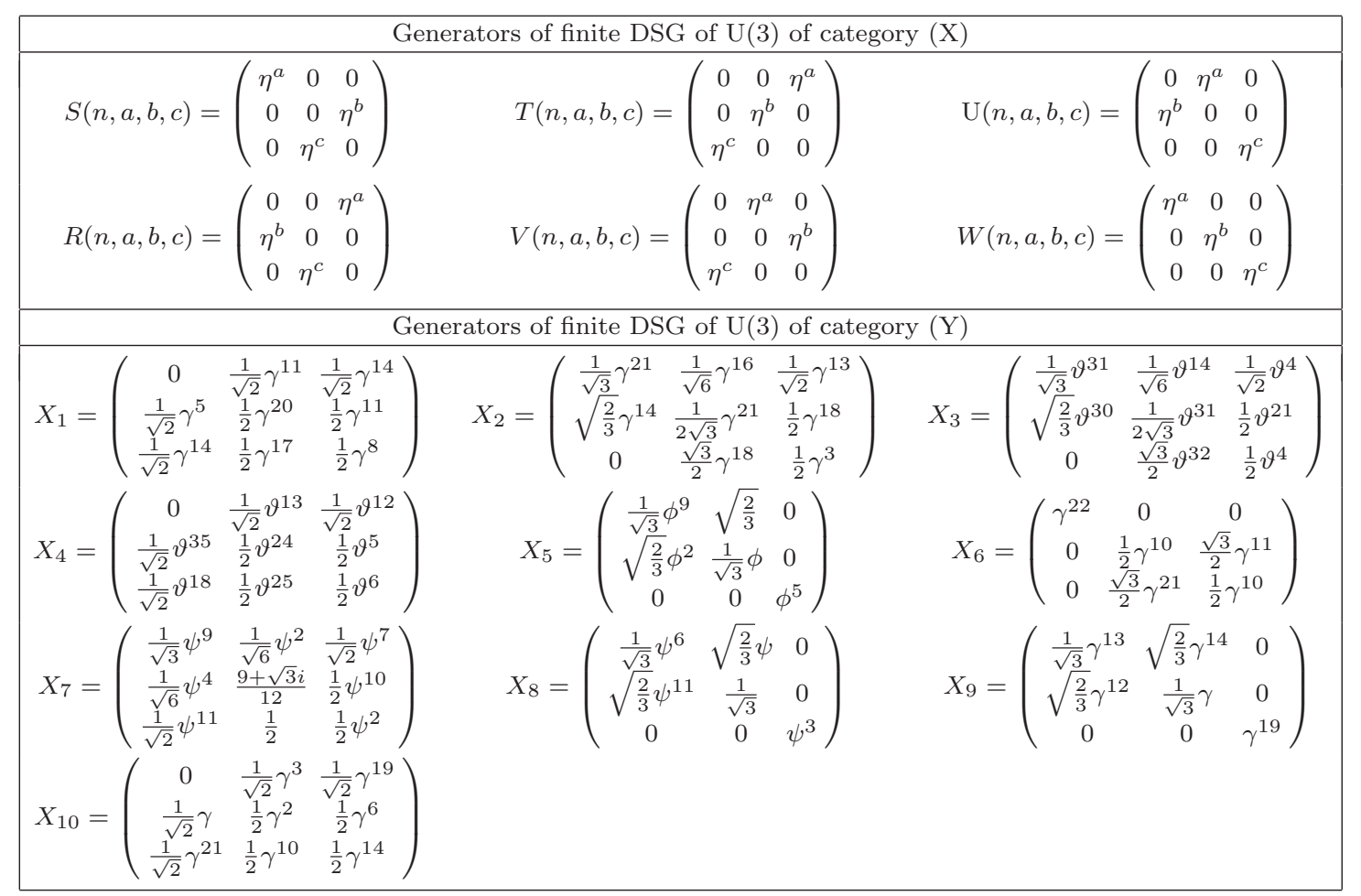

Table 1. Generators of finite DSG of U(3) of order $<512$ as listed in [21]. Here $a, b, c=0,1, \ldots, n-$ $1, \eta=e^{2 \pi i / n}, \gamma=e^{2 \pi i / 24}, \vartheta=e^{2 \pi i / 36}, \phi=e^{2 \pi i / 16}$ and $\psi=e^{2 \pi i / 12}$.

of $T_{l}$ are distinct then the only possible flavour compositions of the massless state are (1) trivial $(1,0,0)$ and its permutation or (2) trimaximal with probability $\frac{1}{3}(1,1,1)$ or (3) BM with probability $\frac{1}{2}(0,1,1)$ or permutations thereof.

Proof. The proof of this theorem follows from few simple observations. We list these and their implications in the following.

(1) Let us define generalized textures with elements in set $\mathcal{H}=\{R, S, T, U, V, W\}$ replaced by $\tilde{\mathcal{H}}=\{\tilde{R}, \ldots, \tilde{W}\}$ obtained by replacing non-zero entries in elements of $\mathcal{H}$ by some arbitrary roots of unity. Thus, for example

$$
\tilde{R} \equiv\left(\begin{array}{ccc}
0 & 0 & \eta_{1} \\
\eta_{2} & 0 & 0 \\
0 & \eta_{3} & 0
\end{array}\right)
$$

with $\left|\eta_{i}\right|=1$. $\mathcal{H}$ is clearly contained in $\tilde{\mathcal{H}}$. It is easy to show that product of any two elements in $\mathcal{H}$ is an element in $\tilde{\mathcal{H}}$, e.g.

$$
R\left(n_{1}, a, b, c\right) \quad R\left(n_{2}, p, q, r\right)=\left(\begin{array}{ccc}
0 & \eta_{1}^{a} \eta_{2}^{r} & 0 \\
0 & 0 & \eta_{1}^{b} \eta_{2}^{p} \\
\eta_{1}^{c} \eta_{2}^{q} & 0 & 0
\end{array}\right) \sim \tilde{V} \in \tilde{\mathcal{H}}
$$

Also product of two elements in $\tilde{\mathcal{H}}$ belongs to $\tilde{\mathcal{H}}$. This implies that all the elements of any group $G_{f}$ generated using elements in $\mathcal{H}$ possesses only one of the six textures 
given in $\tilde{\mathcal{H}}$. It is useful to define subsets $\tilde{\mathcal{H}}_{1}=\{\tilde{W}\}, \tilde{\mathcal{H}}_{2}=\{\tilde{R}, \tilde{V}\}$ and $\tilde{\mathcal{H}}_{3}=\{\tilde{S}, \tilde{T}, \tilde{U}\}$. A product of two elements of $\tilde{\mathcal{H}}_{1}$ belongs to $\tilde{\mathcal{H}}_{1}$ only while a product of any two elements of $\tilde{\mathcal{H}}_{2}$ generates an element which is either in $\tilde{\mathcal{H}}_{1}$ or in $\tilde{\mathcal{H}}_{2}$. A product of any two elements in $\tilde{\mathcal{H}}_{3}$ can belong to either of $\tilde{\mathcal{H}}_{1,2,3} \cdot{ }^{1}$

(2) Since $\tilde{W}$ is diagonal it can either be used as $S_{1 \nu}$ (if one diagonal element is complex and two are unequal and real) or as $T_{l}$ (if all diagonal elements are different). Thus $\tilde{\mathcal{H}}_{1}$ can either be used as a symmetry of the neutrinos or that of the charged leptons.

(3) Eigenvalues of $\tilde{A}=\tilde{R}, \tilde{V}$ are given by

$$
(\operatorname{Det} . \tilde{A})^{\frac{1}{3}}\left(1, \omega, \omega^{2}\right)
$$

with $\omega=e^{2 \pi i / 3}$. Thus at least two eigenvalues are complex and any element of $G_{f}$ with these two structures cannot be used as neutrino symmetry $S_{1 \nu}$. $\tilde{\mathcal{H}}_{2}$ can only be used as symmetry of the charged leptons.

(4) Each element in $\tilde{\mathcal{H}}_{3}$ contains a diagonal and two off-diagonal non-zero entries. Their eigenvalues are of two types. If the off-diagonal entries are complex conjugate of each other then eigenvalues are given by $(\beta, 1,-1)$. This can be used as neutrino symmetry $S_{1 \nu}$ if $\beta \neq \pm 1$. Otherwise elements in $\tilde{\mathcal{H}}_{3}$ also have at least two complex eigenvalues and they would only be suitable as representing $T_{l}$. Thus $\tilde{\mathcal{H}}_{3}$ can be used as a symmetry of the neutrinos and/or charged leptons depending on its structure.

It follows from (2-4) that if flavour group $G_{f}$ belongs to category (X) then there are two possible choices for the neutrino symmetry $S_{1 \nu}$ and three for the the charged lepton symmetry $T_{l}$ apart from their cyclic permutations. We summarize below these choices and matrices which diagonalize them:

(S.a) $S_{1 \nu} \in \tilde{\mathcal{H}}_{1}: S_{1 \nu} \equiv S_{1 \nu}^{a}=\tilde{W}$ and $V_{\nu} \equiv V_{\nu}^{a}=1$.

(S.b) $S_{1 \nu} \in \tilde{\mathcal{H}}_{3}:$ For example,

$$
S_{1 \nu} \equiv S_{1 \nu}^{b}=\left(\begin{array}{ccc}
\beta & 0 & 0 \\
0 & 0 & z^{*} \\
0 & z & 0
\end{array}\right) \text { and } V_{\nu} \equiv V_{\nu}^{b}=\frac{1}{\sqrt{2}}\left(\begin{array}{ccc}
\sqrt{2} & 0 & 0 \\
0 & 1 & -z^{*} \\
0 & z & 1
\end{array}\right)
$$

Other choices of $S_{1 \nu}$ are obtained from the cyclic permutation of above.

(T.a) $T_{l} \in \tilde{\mathcal{H}}_{1}: T_{l} \equiv T_{l}^{a}=\tilde{W}$ and $V_{l} \equiv V_{l}^{a}=1$.

(T.b) $T_{l} \in \tilde{\mathcal{H}}_{2}$ : For example, $T_{l} \equiv T_{l}^{b}=\tilde{R}$ and $V_{l} \equiv V_{l}^{b}=\operatorname{Diag} .\left(1, \eta_{2}, \eta_{1}^{*}\right) U_{\omega}$.

\footnotetext{
${ }^{1}$ The set of above textures is isomorphic to the $S_{3}$ group with $\tilde{W}, \tilde{S}, \tilde{T}, \tilde{U}, \tilde{R}, \tilde{V}$ mapped respectively to elements $e,(23),(13),(12),(132),(123)$ of $S_{3}[5]$.
} 
(T.c) $T_{l} \in \tilde{\mathcal{H}}_{3}:$ For example,

$$
T_{l} \equiv T_{l}^{c}=\left(\begin{array}{ccc}
\beta & 0 & 0 \\
0 & 0 & z_{1} \\
0 & z_{2} & 0
\end{array}\right) \text { and } V_{l} \equiv V_{l}^{c}=\frac{1}{\sqrt{2}}\left(\begin{array}{ccc}
\sqrt{2} & 0 & 0 \\
0 & 1 & -\left(z_{1} z_{2}^{*}\right)^{\frac{1}{2}} \\
0 & \left(z_{2} z_{1}^{*}\right)^{\frac{1}{2}} & 1
\end{array}\right)
$$

where $\tilde{R}$ is defined in eq. (3.1) and

$$
U_{\omega}=\frac{1}{\sqrt{3}}\left(\begin{array}{ccc}
1 & 1 & 1 \\
1 & \omega^{2} & \omega \\
1 & \omega & \omega^{2}
\end{array}\right)
$$

$\omega=e^{2 \pi i / 3}$. Other choices of $T_{l}$ are obtained from the cyclic permutation of above.

The above alternatives for $S_{1 \nu}$ and $T_{l}$ determine complete mixing pattern (apart from cyclic permutations) which is possible for all the flavour groups belonging to category (X). The detailed values of the mixing angles depend upon the values of complex parameters appearing above but flavour content of the massless state in all the cases is not sensitive to them. To see this, note that eigenstate of $S_{1 \nu}$ corresponding to a massless neutrino for all the choices listed above is given uniquely by $(1,0,0)^{T}$ or its cyclic permutations and let us consider $\left|\psi_{0}\right\rangle=(0,0,1)^{T}$ for definiteness. The corresponding flavour state in the basis with diagonal $T_{l}$ (and hence diagonal charged lepton mass matrix $M_{l} M_{l}^{\dagger}$ ) is given by $|\psi\rangle=V_{l}^{\dagger}\left|\psi_{0}\right\rangle$. Then three possible choices for $V_{l}$ listed above give three flavour mixing mentioned in the theorem stated above. (a) $V_{l}=V_{l}^{a}$ implies an unmixed massless $|\psi\rangle$ with probabilities $(0,0,1)$ or (b) $V_{l}=V_{l}^{b}$ implies trimaximally mixed $|\psi\rangle$ with probabilities $\frac{1}{3}(1,1,1)$ or $(\mathrm{c}) V_{l}=V_{l}^{c}$ implies bimaximally mixed $|\psi\rangle$ with probabilities $\frac{1}{2}(0,1,1)$. Other choices allowed by permutations of the chosen $S_{1 \nu}$ and $T_{l}$ only permute the above mentioned flavour compositions.

The above result has strong phenomenological implications due to the fact that the structure of the massless state determines one column of the mixing matrix. If only one neutrino is massless then the massless state has to be identified with the first (third) column of the neutrino mixing matrix in the standard convention in case of the normal (inverted) hierarchy. Then, for the trimaximal structure, one predicts $\sin ^{2} \theta_{13}=\frac{1}{3}$ for the inverted and $\cos ^{2} \theta_{13} \cos ^{2} \theta_{12}=\frac{1}{3}$ for the normal hierarchy. Both of these differ from the experimental values and would need large corrections. The BM composition is more suitable to describe inverted hierarchy and taken as the third column of $U_{\mathrm{PMNS}}$ would predict $\theta_{23}=\frac{\pi}{4}$ and $\theta_{13}=0$. These predictions signaling $\mu-\tau$ symmetry have often been considered as a good zeroth order ansatz which can follow in a large number of groups in category (X) as we will show. But with either choice, one does not get the correct leading order value for the solar mixing angle in the whole class of groups in the category (X).

\subsection{Mixing patterns with a massless neutrino}

The foregoing discussion also allows us to determine all possible mixing matrices for all the groups in category $(\mathrm{X})$. We list below non-trivial choices. 
(1) If $S_{1 \nu} \in \tilde{\mathcal{H}}_{1}$ and $T_{l} \in \tilde{\mathcal{H}}_{2}$ then $U_{\mathrm{PMNS}}$ has democratic structure:

$$
\left|U_{\mathrm{PMNS}}\right|=\frac{1}{3}\left(\begin{array}{lll}
1 & 1 & 1 \\
1 & 1 & 1 \\
1 & 1 & 1
\end{array}\right)
$$

The above structure which can be realized for both normal and inverted hierarchy implies $\sin ^{2} \theta_{13}=\frac{1}{3}, \sin ^{2} \theta_{12}=\sin ^{2} \theta_{23}=\frac{1}{2}$. It fails to predict two of the mixing angles correctly at the leading order and would need very large corrections.

(2) If $S_{1 \nu}$ is to be non-diagonal then it is given by the choice (S.b). With $T_{l}=T_{l}^{b}$ as in (T.b), one gets $U_{\mathrm{PMNS}}$ as

$$
U_{\mathrm{PMNS}}=\frac{1}{\sqrt{6}}\left(\begin{array}{ccc}
\sqrt{2} & p \eta_{2}^{*}+p^{*} \eta_{1} z^{*} & z\left(p^{*} \eta_{1} z^{*}-p \eta_{2}^{*}\right) \\
\sqrt{2} & p \eta_{2}^{*} \omega+p^{*} \eta_{1} z^{*} \omega^{2} & z\left(p^{*} \eta_{1} z^{*} \omega^{2}-p \eta_{2}^{*} \omega\right) \\
\sqrt{2} & p \eta_{2}^{*} \omega^{2}+p^{*} \eta_{1} z^{*} \omega & z\left(p^{*} \eta_{1} z^{*} \omega-p \eta_{2}^{*} \omega^{2}\right)
\end{array}\right)
$$

where, $p=\left(\eta_{1} \eta_{2} \eta_{3}\right)^{\frac{1}{3}}$. Note that the first column determining massless state is fixed for the normal hierarchy while one can permute the second and third columns by interchanging the order of the two real eigenvalues of $S_{1 \nu}$. Similarly, changing the order of the three rows amount to reordering the eigenvalues of $T_{l}$. Thus there are six possible choices for the reactor mixing angle $\theta_{13}$ and minimum of this should be identified with $\sin ^{2} \theta_{13}$ and the corresponding column as the third column of the PMNS matrix. Explicitly,

$$
\sin ^{2} \theta_{13}=\frac{1}{3} \operatorname{Min} .\left|1 \pm \operatorname{Re}\left(\eta_{3}^{*} p^{*} \tilde{\lambda}_{i} z\right)\right|,
$$

where \pm sign correspond to two columns and rows are labeled by

$$
\tilde{\lambda}_{i}=\left(1, \omega, \omega^{2}\right) \text {. }
$$

$\eta_{3}$ is defined in eq. (3.1). The $\sin ^{2} \theta_{23}$ also gets determined by the entries in the same column as that of $\sin ^{2} \theta_{13}$. Moreover, it is seen from eq. (3.4) that

$$
\operatorname{Im}\left(U_{12} U_{13}^{*} U_{23} U_{22}^{*}\right)=0
$$

independent of the values of complex parameters showing that all the groups in category (X) lead to the absence of the Dirac CP violation even if they predict non-zero $\theta_{13}$. Alternative choice $T_{l}=\tilde{V}$ also give prediction similar to above but other possible choices of $T_{l}$, namely $T_{l} \in \tilde{\mathcal{H}}_{3}$ give unacceptable predictions for mixing angles in case of the normal hierarchy.

(3) Unique choice for $S_{1 \nu}$ in case of the inverted hierarchy is given by

$$
S_{1 \nu}=\tilde{U}=\left(\begin{array}{ccc}
0 & z_{\nu} & 0 \\
z_{\nu}^{*} & 0 & 0 \\
0 & 0 & \beta_{\nu}
\end{array}\right) .
$$


The corresponding choice for $T_{l}$ giving non-trivial pattern is given by $T_{l}^{c}$ in (T.c). The resulting mixing matrix is given by:

$$
U_{\mathrm{PMNS}}=\left(\begin{array}{ccc}
\frac{1}{\sqrt{2}} & -\frac{z_{\nu}}{\sqrt{2}} & 0 \\
\frac{z_{\nu}^{*}}{2} & \frac{1}{2} & \frac{\left(z_{2} z_{1}^{*}\right)^{\frac{1}{2}}}{\sqrt{2}} \\
-\frac{z_{\nu}^{*}\left(z_{1} z_{2}^{*}\right)^{\frac{1}{2}}}{2} & -\frac{\left(z_{1} z_{2}^{*}\right)^{\frac{1}{2}}}{2} & \frac{1}{\sqrt{2}}
\end{array}\right)
$$

Note that the $\left|U_{\text {PMNS }}\right|$ has the $\mathrm{BM}$ values $\theta_{12}=\theta_{23}=\frac{\pi}{4}$ and $\theta_{13}=0$ independent of the values of the group dependent complex parameters. In this sense, the BM mixing pattern is more universal and follows in many groups constructed from the category $(\mathrm{X})$.

The case (2) discussed above can lead to reasonable values for the mixing angles $\theta_{13}$, $\theta_{23}$ and we will explore various possibilities numerically in the next section. The $\theta_{12}$ is predicted either $\theta_{12}=\frac{\pi}{4}$ or close to $\theta_{12} \sim 54^{\circ}$. This needs to be changed and we shall discuss possible ways to modify this in section 5 .

Before closing this section, we wish to emphasize generality of the result derived here. Firstly, this result is valid for any group $G_{f}$ in category (X) independent of its order. Secondly, large number of the finite DSG of U(3) fall in category (X) to which the theorem derived here is applicable. In addition to 70 of the 75 groups with order $<512$, six infinite series of groups also fall in this category. These are generated by the following specific matrices of table 1:

$$
\begin{aligned}
\Sigma\left(3 n^{3}\right) & \equiv\{W(n, 0,0,1), W(n, 1,0,0), E\} ; \\
T_{N}(m) & \equiv\left\{e^{2 \pi i / 3^{m}} E, W(n, 1, a, n-1-a)\right\}, \quad\left(1+a+a^{2}\right) \bmod n=0 ; \\
S_{4}(m) & \equiv\left\{S\left(2^{m}, 1,1+2^{m-1}, 1\right), T\left(2^{m}, 3,3,2^{m-1}+3\right)\right\} ; \\
\Delta\left(3 n^{2}, m\right) & \equiv\left\{e^{2 \pi i / 3^{m}} E, W(n, 0,1, n-1)\right\} ; \\
\Delta\left(6 n^{2}, m\right) & \equiv\left\{E, W(n, 0,1, n-1), T\left(2^{m}, 1+2^{m-1}, 1+2^{m-1}, 1+2^{m-1}\right)\right\} ; \\
\Delta\left(6 n^{2}, j, k\right) & \equiv\left\{E, W(n, 0,1, n-1), T\left(3^{j} 2^{k}, 1+3^{j} 2^{k-1}, 1+3^{j} 2^{k-1}, 1+3^{j} 2^{k-1}\right)\right\} .
\end{aligned}
$$

$E$ defined above is a matrix with determinant 1 and can be written as $E=V(p, 0,0,0)$ independent of $p$. It is clear from the structure of the generators that the group series $\Sigma\left(3 n^{3}\right), T_{n}(m), \Delta\left(3 n^{2}, m\right)$ either contain diagonal elements or elements with two complex eigenvalues. Thus these groups provide an example of the case (1) discussed before eq. (3.3) and only mixing pattern possible within these groups is democratic mixing. Of these, $\Sigma\left(3 n^{3}\right)$ were already discussed in [20].

The above theorem does not however hold for the groups in category (Y). These are not easily amenable to analytic discussion but we study them numerically and show that one of these can give quite satisfactory leading order predictions for the mixing angles. 


\section{A numerical scan of discrete subgroups of $\mathrm{U}(3)$}

We now study numerically the predicted values of the reactor and atmospheric mixing angles for all the DSG of U(3) of order $<512$. These include all the 70 groups of category $(\mathrm{X})$ and 5 groups of category $(\mathrm{Y})$. It is clear form the discussions in the last section that if the groups of category $(\mathrm{X})$ are used as flavour symmetry for a massless neutrino then sizable corrections at least in the solar angle would be needed which in general may change the other angles also. It is thus appropriate to work out leading order predictions for other angles. While the predicted solar angle is almost universal for all the groups under study, the other angles depend on the details of the group and we present this numerically. In our analysis, we choose a particular group and look at its suitability to be a flavour symmetry with a massless neutrino and work out all possible values of the predicted mixing angles.

\subsection{Results of the groups in category $(\mathrm{X})$}

First, we restrict ourselves to the groups of category $(\mathrm{X})$ and of order $<512$ tabulated by Ludl [21]. We demand that (1) this groups should contain at least one element to be identified as $S_{1 \nu}$ with eigenvalue $(\eta, 1,-1)$ with $\eta^{N}=1$ and $N \geq 3$ appropriate to describe a massless neutrino, (2) $T_{l}$ should have all three different eigenvalues and (3) $S_{1 \nu}$ does not commute with $T_{l}$. These requirements are essential but quite restrictive and rule out large number of groups as possible $G_{f}$. Consider all the groups generated only from the matrices $R, V$ and $W$ alone for arbitrary values of the integers defining these matrices. As mentioned in the last section, the above structures close among themselves as result the groups in question contain either diagonal elements or elements with at least two complex eigenvalues. The neutrino symmetry in this case would come only from the diagonal elements and one would get only democratic mixing which is not of much interest. There are 48 such groups among the 70 listed groups and the same argument would apply to any higher order groups generated using any of these three matrices. Requirement (1) is also restrictive and we find numerically that of the remaining 22 groups, 16 get ruled out by this requirement. This leaves only six groups which can be used as suitable flavour symmetry. We numerically find all the elements which can be used as $S_{1 \nu}$ and $T_{l}$ within these allowed groups and determine the resulting mixing matrix. The allowed groups and predicted values of the mixing angles are listed in table 2 .

We have restricted ourselves only to choices which give $\sin ^{2} \theta_{13} \leq(0.25)^{2}$. In this case one gets only four possible forms of $\left|U_{\mathrm{PMNS}}\right|$ in case of the normal neutrino mass hierarchy. They are listed as $M_{1}, M_{2}, M_{3}, M_{4}$ below:

$$
M_{1}=\left(\begin{array}{ccc}
0.5774 & 0.7887 & 0.2113 \\
0.5774 & 0.5774 & 0.5774 \\
0.5774 & 0.2113 & 0.7887
\end{array}\right) ; \quad M_{2}=\left(\begin{array}{ccc}
0.5774 & 0.8041 & 0.1418 \\
0.5774 & 0.5248 & 0.6255 \\
0.5774 & 0.2793 & 0.7673
\end{array}\right)
$$




\begin{tabular}{|cccccc|}
\hline Group & Classification & Generators & $\left|U_{\mathrm{PMNS}}\right|$ & $\sin ^{2} \theta_{13}$ & $\sin ^{2} \theta_{23}$ \\
\hline$\llbracket 48,30 \rrbracket$ & $S_{4}(2)$ & $S(4,1,3,1), T(4,3,3,1)$ & $M_{1}$ & 0.0447 & 0.349 \\
$\llbracket 162,10 \rrbracket$ & & $S(3,0,1,0), T(3,1,1,0)$ & $M_{2}$ & 0.0201 & 0.399 \\
& & & $M_{3}$ & 0 & 0.5 \\
$\llbracket 192,182 \rrbracket$ & $\Delta\left(6 \cdot 4^{2}, 2\right)$ & $T(4,0,2,1), \mathrm{U}(4,3,0,2)$ & $M_{1}$ & 0.0447 & 0.349 \\
& & & $M_{3}$ & 0 & 0.5 \\
$\llbracket 384,571 \rrbracket$ & $\Delta\left(6 \cdot 4^{2}, 3\right)$ & $T(8,1,5,3), \mathrm{U}(8,1,3,7)$ & $M_{4}$ & 0.0114 & 0.424 \\
$\llbracket 432,260 \rrbracket$ & $\Delta\left(6 \cdot 6^{2}, 2\right)$ & $S(12,7,5,3), T(12,9,5,7), \mathrm{U}(12,9,1,11)$ & $M_{1}$ & 0.0447 & 0.349 \\
$\llbracket 486,125 \rrbracket$ & & $S(9,2,5,2), T(9,7,7,4), \mathrm{U}(3,0,1,1)$ & $M_{2}$ & 0.0201 & 0.399 \\
& & & $M_{3}$ & 0 & 0.5 \\
\hline
\end{tabular}

Table 2. Predictions for $\theta_{23}$ and $\theta_{13}$ for the normal hierarchy $\left(m_{\nu_{1}}=0\right)$ from a scan of the DSG of $\mathrm{U}(3)$ of category $(\mathrm{X})$ and order $<512$. The group identity $\llbracket g, j \rrbracket$ denotes the $j^{\text {th }}$ finite group of order $g$ as classified in the Small Group Library of the computer algebra system GAP [28]. The prediction for $\sin ^{2} \theta_{23}$ is chosen to be in the first octant of $\theta_{23}$. The structures $M_{1}-M_{4}$ for $U_{\mathrm{PMNS}}$ are specified in eq. (4.1).

$$
M_{3}=\left(\begin{array}{lll}
\sqrt{\frac{1}{3}} & \sqrt{\frac{2}{3}} & 0 \\
\sqrt{\frac{1}{3}} & \sqrt{\frac{1}{6}} & \sqrt{\frac{1}{2}} \\
\sqrt{\frac{1}{3}} & \sqrt{\frac{1}{6}} & \sqrt{\frac{1}{2}}
\end{array}\right) ; \quad \quad M_{4}=\left(\begin{array}{ccc}
0.5774 & 0.8095 & 0.1066 \\
0.5774 & 0.4971 & 0.6478 \\
0.5774 & 0.3125 & 0.7543
\end{array}\right)
$$

Note that there exist two predictions for $\theta_{23}$ in each of the above pattern as the second and third row can be interchanged though in table 2 we select its first octant $\left(\theta_{23} \leq \pi / 4\right)$ values as they are favored at $2 \sigma$ in case of normal hierarchy by a recent global fit [29]. It is seen from the above equations that

- Structure $M_{3}$ displays $\mu-\tau$ symmetry.

- $M_{4}$ predicts $\sin ^{2} \theta_{23}$ close to the best fit value as per the global fits in [29]. The predicted $\sin ^{2} \theta_{13}$ requires significant corrections.

- $M_{2}$ fares better and predicts $\sin ^{2} \theta_{13}$ within $2 \sigma$ and $\sin ^{2} \theta_{23}$ within $1 \sigma$ of their best fit values.

All the structures have trimaximally mixed massless state and predict $\sin ^{2} \theta_{12} \sim \frac{2}{3}$. Situation changes if one were to impose lepton number as an additional symmetry and assume that neutrinos are Dirac particles. All the residual symmetries considered here and many more will be allowed choices in this case but now none of the neutrinos would be forced to be massless. In this case, one has the freedom to interchange the first and the second columns of all the structures obtained here. The group $\llbracket 162,10 \rrbracket$ in this case will predict all the mixing angles correctly within $3 \sigma$. This group has already been identified as a possible group for the Dirac neutrinos [30].

The above results are obtained for the normal hierarchy. The inverted hierarchy allows only the BM pattern as argued analytically in the previous section. All the groups listed in table 2 predicts BM mixing for the inverted hierarchy. The group $S_{4}(2)$ is the smallest among them and has been studied in detail in [20] where an explicit model giving satisfactory mixing pattern is discussed. 


\subsection{Results of the groups in category $(\mathrm{Y})$}

We now discuss the groups in category $(\mathrm{Y})$ with order $<512$ listed by Ludl [21]. There are only five such groups having 3-dimensional faithful IR. The corresponding generators are given by matrices $X_{1}-X_{10}$ and reproduced here in table 1 . Consecutive pairs of matrices $X_{i}, X_{i+1}$ with $i=1,3,5,7,9$ generate groups of order 216, 324, 432, 432, 432 respectively. We have numerically generated all the elements of these five groups and find that four of the five groups do not contain any element with one complex and two real eigenvalues. They therefore do not qualify as possible flavour groups for a massless neutrino in this

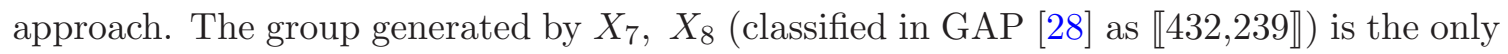
one which contain such elements and we analyze it further.

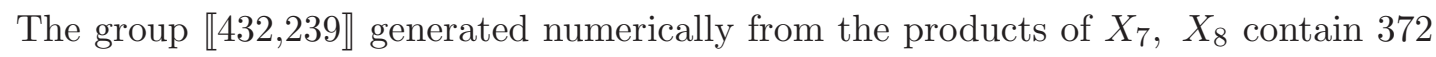
elements with all eigenvalues unequal. Of these, 72 have two real eigenvalues and would be appropriate as a member of $G_{\nu}$. The corresponding charged lepton symmetry group $G_{l}$ may be generated from any of the 372 elements. Taking only the non-commutative set of $G_{l}$ and $G_{\nu}$, all the mixing angles are fully predicted with any of these choices due to their completely different eigenvalues. We have numerically constructed all possible mixing matrices using elements in these sets. We find that this group allows only two possible structures for the massless state. These have flavour content $\sim(0.59,0.25,0.16)$ or $(0,1 / 2 /, 1 / 2)$ and permutations of these. Since the former now differs from the trimaximal structure predicted in class (X) groups, there is a possibility of better agreement with the data. We explore it further by demanding that the reactor mixing angle should satisfy $\left|\sin \theta_{13}\right| \leq 0.25$. It turns out that only two possible mixing structures satisfy this restriction, one for the normal and one for the inverted hierarchy. These are respectively given by,

$$
\left|U_{\text {PMNS }}\right|=\left(\begin{array}{ccc}
0.7691 & 0.6124 & 0.183 \\
0.3981 & 0.683 & 0.6124 \\
0.5 & 0.3981 & 0.7691
\end{array}\right) \text { for normal hierarchy }
$$

and

$$
\left|U_{\text {PMNS }}\right|=\left(\begin{array}{ccc}
0.8881 & 0.4597 & 0 \\
0.3251 & 0.628 & 0.7071 \\
0.3251 & 0.628 & 0.7071
\end{array}\right) \text { for inverted hierarchy. }
$$

The predicted mixing angles are:

$$
\begin{array}{lll}
\sin ^{2} \theta_{12}=0.388, & \sin ^{2} \theta_{23}=0.388, & \sin ^{2} \theta_{13}=0.0335 \text { for normal hierarchy }, \\
\sin ^{2} \theta_{12}=0.211, & \sin ^{2} \theta_{23}=1 / 2, & \sin ^{2} \theta_{13}=0 \text { for inverted hierarchy } .
\end{array}
$$

The prediction in case of the normal hierarchy does not reproduce all the mixing angles within $3 \sigma$. But it is fairly close to the observed ones with $\chi^{2} \sim 45$. It is thus conceivable that small non-leading order effect can correct the predictions. The same predictions can be obtained for all massive neutrinos if they are of Dirac type. ${ }^{2}$ The predictions in case of the

\footnotetext{
${ }^{2}$ The same pattern is obtained for Dirac neutrinos in [31] using the group $\Sigma(36 \times 3)$ which is an $\mathrm{SU}(3)$

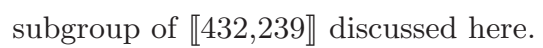


inverted hierarchy are similar in some sense to the BM mixing. The predicted $\theta_{23}$ and $\theta_{13}$ are the same as in the BM mixing. The solar angle predicted here requires large corrections which are similar in magnitude to the case of the BM mixing but now in opposite direction.

The above mixing matrices can arise with several different choices of $S_{1 \nu}$ and $T_{l}$ from elements of the group. We give here an example for the normal hierarchy which happens to be the simplest choice:

$$
\begin{gathered}
T_{l}=X_{7} X_{8}=\left(\begin{array}{ccc}
\frac{i}{3}+\frac{1}{3} e^{\frac{i \pi}{6}} & -\frac{1}{3} i \sqrt{2} e^{\frac{i \pi \pi}{6}}+\frac{e^{\frac{i \pi}{3}}}{3 \sqrt{2}} & \frac{i e^{-\frac{5 i \pi}{6}}}{\sqrt{2}} \\
\frac{(9+i \sqrt{3}) e^{-\frac{i \pi}{6}}}{6 \sqrt{6}}-\frac{e^{\frac{2 i \pi}{3}}}{3 \sqrt{2}} & \frac{9+i \sqrt{3}}{12 \sqrt{3}}+\frac{1}{3} e^{\frac{5 i \pi}{6}} & \frac{1}{2} i e^{-\frac{i \pi}{3}} \\
0 & \frac{\sqrt{3}}{2} & \frac{1}{2} i e^{\frac{i \pi}{3}}
\end{array}\right) \\
S_{1 \nu}=X_{8} X_{7}=\left(\begin{array}{ccc}
\frac{i}{3}+\frac{1}{3} e^{\frac{5 i \pi}{6}} & \frac{(9+i \sqrt{3}) e^{\frac{i \pi}{6}}}{6 \sqrt{6}}-\frac{e^{\frac{i \pi}{3}}}{3 \sqrt{2}} & \frac{e^{\frac{i \pi}{6}}}{\sqrt{6}}-\frac{e^{-\frac{5 i \pi}{6}}}{\sqrt{6}} \\
-\frac{1}{3} i \sqrt{2} e^{-\frac{i \pi}{6}}+\frac{e^{\frac{2 i \pi}{3}}}{3 \sqrt{2}} & \frac{9+i \sqrt{3}}{12 \sqrt{3}}+\frac{1}{3} e^{\frac{i \pi}{6}} & -\frac{1}{\sqrt{3}}+\frac{e^{-\frac{i \pi}{3}}}{2 \sqrt{3}} \\
\frac{i e^{-\frac{i \pi}{6}}}{\sqrt{2}} & \frac{i}{2} & \frac{1}{2} i e^{\frac{i \pi}{3}}
\end{array}\right)
\end{gathered}
$$

Both $S_{1 \nu}$ and $T_{l}$ have identical set of eigenvalues $(i, 1,-1)$ and each therefore generate a $Z_{4}$ subgroups of the full group. Diagonalization of $S_{1 \nu}$ and $T_{l}$ generates the mixing matrix given in eq. (4.2). One also finds trivial $\mathrm{CP}$ phase and therefore absence of $\mathrm{CP}$ violation in neutrino oscillations.

\section{Modifying solar mixing angle: two alternatives}

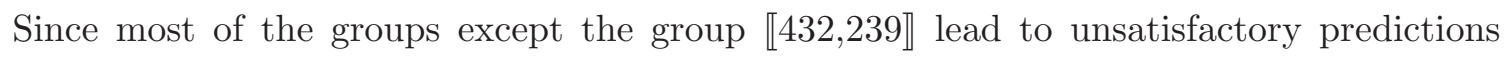
for the solar mixing angle, it is appropriate to explore possibilities which allows one to replace or avoid such prediction. We propose two alternatives, (1) use of discrete groups admitting reducible $(2+1)$-dimensional faithful representation such that one of the three mixing angles remains unpredicted and (2) modifications in the neutrino mass matrices predicted at the leading order in groups in class $(\mathrm{X})$.

\subsection{Discrete subgroups of $\mathrm{U}(2) \times \mathrm{U}(1)$}

We now depart from the requirement of assigning leptons to 3 -dimensional IR and consider instead alternative possibility of putting them into a reducible $(2+1)$-dimensional faithful representation. Such groups will be subgroup of $\mathrm{U}(2) \times \mathrm{U}(1)[21,22]$. As we will see, consideration of such groups allows elements having the structure of $S_{1 \nu}^{\prime}$, eq. (2.6) with two degenerate eigenvalues. One mixing angle corresponding to rotation in the plane of the degenerate eigenvalues remain undetermined in this case. Thus this scheme is less predictive but can avoid wrong prediction of the solar mixing angle.

Let us consider again the set of groups generated from the matrices in table 1. One would generate subgroups of $\mathrm{U}(2) \times \mathrm{U}(1)$ from this set if they are generated from block- 
diagonal matrices only. Thus combination of two different $S$ or $S$ and $W$ would ${ }^{3}$ generate such groups and the same would apply when $S$ is replaced by $T$ or $U$. There exist three different possibilities for choosing neutrino and charged lepton symmetries from the elements of the group generated this way. (a) All the eigenvalues of the neutrino and the charged lepton symmetry generators are different, (b) two of the real eigenvalues of the neutrino symmetry are degenerate and the charged lepton symmetry has three distinct eigenvalues and (c) neutrino symmetry is characterized by different eigenvalues and the charged lepton symmetry has two degenerate eigenvalues. It is easy to see that possibility (a) predicts two of the mixing angles to be zero. In case of possibility (b) only reasonable mixing pattern is found with the choice

$$
S_{1 \nu}^{\prime}=\operatorname{Diag} .(1,1, \eta) \quad \text { and } \quad T_{l}=\left(\begin{array}{ccc}
\eta_{1} & 0 & 0 \\
0 & 0 & \eta_{2} \\
0 & \eta_{3} & 0
\end{array}\right) .
$$

This case corresponds to the inverted hierarchy and the mixing pattern given by

$$
U_{\mathrm{PMNS}}=U_{23}(\pi / 4) U_{12}(\theta),
$$

where $U_{i j}(\theta)$ is a unitary rotation in the $i j$-plane with an angle $\theta$ and undetermined phases. The above mixing matrix corresponds to the $\mu-\tau$ symmetric structure and predict $\theta_{13}=0$ and $\theta_{23}=\pi / 4$. The small next to leading order effects are known to generate large enough $\theta_{13}$ in this case [32]. In case (c), the atmospheric mixing angle remains undetermined and the solar angle gets fixed to $\pi / 4$.

The $S_{1 \nu}^{\prime}$ and $T_{l}$ given above close to a finite group if $\eta, \eta_{1,2,3}$ all are $n^{\text {th }}$ roots of unity. $S_{1 \nu}^{\prime}$ is given in this case by appropriate $W$ in table 1 and $T_{l}$ by $S$. Now define three generators:

$$
A=\left(\begin{array}{ccc}
\eta & 0 & 0 \\
0 & 1 & 0 \\
0 & 0 & 1
\end{array}\right) ; \quad B=\left(\begin{array}{ccc}
1 & 0 & 0 \\
0 & 0 & 1 \\
0 & 1 & 0
\end{array}\right) ; \quad C=\left(\begin{array}{ccc}
1 & 0 & 0 \\
0 & \eta & 0 \\
0 & 0 & 1
\end{array}\right)
$$

where $\eta=e^{2 \pi i / n}$. The elements in the set $\mathcal{A}=\left\{S(n, a, b, c), W\left(n, a^{\prime}, b^{\prime}, c^{\prime}\right)\right\}$ can be written in terms of $A, B, C$ as:

$$
S(n, a, b, c)=A^{a} C^{b} B C^{c} ; \quad W\left(n, a^{\prime}, b^{\prime}, c^{\prime}\right)=A^{a^{\prime}} C^{b^{\prime}}(B C B)^{c^{\prime}}
$$

Thus $S$ and $W$ in the set $\mathcal{A}$ are elements of the group generated by $A, B, C$. It is known that $B, C$ generate the group $\Sigma\left(2 n^{2}\right)[5,20]$. Also $A$ commutes with $B, C$ and they all together generate the group $\Sigma\left(2 n^{2}\right) \times Z_{n}$. It follows therefore that the residual symmetries in eq. (5.1) close to a subgroup of $\Sigma\left(2 n^{2}\right) \times Z_{n}$ which may be considered as appropriate flavour group corresponding to a massless neutrino and $\mu-\tau$ symmetry. Such groups without the factor $Z_{n}$ were considered as possible symmetry for the massless neutrino in [20]. The

\footnotetext{
${ }^{3}$ note that product of two $S$ generate a $W$ like structure or product of $S, W$ generate a structure similar to $S$.
} 
$Z_{n}$ factor is not required if $\eta_{2} \eta_{3} \neq 1$ since in this case, all the eigenvalues of $T_{l}$ can be different even when $\eta_{1}=1$. As the above argument shows, the group series $\Sigma\left(2 n^{2}\right)$ or even their subgroups in some cases may also be sufficient to harness appropriate residual symmetry. Let us give some examples:

$\begin{array}{ccc}\text { Generators } & \text { Order } & S_{\nu}^{\prime} \\ S(3,0,1,1), W(3,0,0,1) & 18 & W \\ S(4,0,1,1), W(4,0,0,1) & 32 & W \\ S(6,0,4,3), W(6,0,3,1) & 36 & W^{2} \\ S(12,0,4,2), W(12,0,4,1) & 96 & W^{3}\end{array}$

The $S$ given in above examples can be considered as generating $G_{l}$ and powers of $W$ mentioned in the last column can be used $S_{1 \nu}^{\prime}$. In the list above, the first two groups are $\Sigma\left(2 \cdot 3^{2}\right)$ and $\Sigma\left(2 \cdot 4^{2}\right)$ while the last two are subgroups of $\Sigma\left(2 \cdot 6^{2}\right)$ and $\Sigma\left(2 \cdot 12^{2}\right)$ respectively.

\subsection{Modified neutrino mass matrices}

All the results derived so far are valid at the leading order when the flavour symmetry group $G_{f}$ is suitably broken into $G_{l}$ and $G_{\nu}$ which imply a unique contribution to the charged lepton and neutrino mass matrices. One can however consider a more general possibility in which either the charged lepton and/or the neutrino mass matrices contain two separate pieces, each emerging separately after the spontaneous symmetry breaking of $G_{f}$. The second piece may also be a leading order effect arising from a set of flavons with different vacuum configurations or may represent perturbation to the leading order predictions arising from the next to leading order operators which do not respect $G_{l}$ or $G_{\nu}$ The prediction of a massless neutrino will be maintained if $G_{\nu}$ remains intake and both these corrections emerge in the charged lepton sector only. We have already discussed one such example in the context of specific $S_{4}(2)$ model in [20] with inverted hierarchy and BM mixing pattern. In this example, $S_{4}(2)$ is broken down to $G_{\nu}=Z_{4}$ in the neutrino sector while it is completely broken in the charged lepton sector leaving an accidental $Z_{2}$ symmetry, see [20] for more details. It is found that such a correction modifies both the solar and the reactor mixing angles and brings all three mixing angles in agreement with their global fit values at $2 \sigma$ ranges.

Here we consider a scenario in which neutrino mass matrix consist of two pieces each invariant under separate symmetries contained in $G_{f}$ :

$$
M_{\nu}=M_{1 \nu}+M_{2 \nu} .
$$

Both $M_{i \nu},(i=1,2)$ can arise from different sets of flavons after spontaneous breaking of $G_{f}$ and each is assumed to be characterized by its own residual symmetries

$$
S_{i}^{T} M_{i \nu} S_{i}=M_{i \nu}
$$

The combined residual symmetry of the neutrino mass matrix need not be either of $S_{i}$ but the form of each $M_{i}$ is restricted by eq. (5.5) and still a predictive model which can 
correct for the wrong solar mixing angle can emerge from such breaking of $G_{f}$ as we shall illustrate through two different examples, one for the normal and the other for the inverted hierarchy. If $S_{1}$ and $S_{2}$ commute then the leading order prediction for the mixing angles would remain unaffected but the combined effect of two pieces would be to change the mass ordering which can lead to correct mixing angle predictions. Both mixing angles and mass ordering can change when $S_{1}$ and $S_{2}$ do not commute. The following two examples illustrate these two possibilities.

\subsubsection{The group $\llbracket 162,10 \rrbracket$}

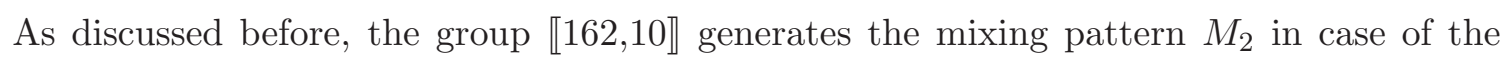
normal hierarchy. This predicts viable values of $\theta_{13}$ and $\theta_{23}$. A satisfactory prediction of the solar mixing angle can be obtained if the first and second columns of $M_{2}$ can be interchanged. However, this interchange leads to an inconsistent prediction $m_{\nu_{2}}=0$. This can be corrected by admitting a second piece in $M_{\nu}$.

An example of the residual symmetries of neutrinos and charged leptons which give rise to the pattern $M_{2}$ in group $\llbracket 162,10 \rrbracket$ is given by

$$
S_{1}=\left(\begin{array}{ccc}
\omega & 0 & 0 \\
0 & 0 & \omega \\
0 & \omega^{2} & 0
\end{array}\right) \quad \text { and } \quad T_{l}=\left(\begin{array}{ccc}
0 & \omega^{2} & 0 \\
0 & 0 & 1 \\
1 & 0 & 0
\end{array}\right)
$$

with $\omega=e^{2 \pi i / 3}$. The neutrino mass matrix invariant under $S_{1}$ takes the form

$$
M_{1 \nu}=\left(\begin{array}{ccc}
0 & 0 & 0 \\
0 & x & c \\
0 & c & \omega^{2} x
\end{array}\right),
$$

where $x$ and $c$ are complex parameters. The above matrix is diagonalized by $V_{\nu}^{b}$ given in (S.b) in section 3.1 with a replacement of $z$ by $\omega$.

One of the eigenvalues in lower $2 \times 2$ block of $M_{1 \nu}$ vanishes if an ad hoc condition $c= \pm \omega x$ is imposed. This leads to two massless neutrinos in $M_{1 \nu}$ and $m_{\nu_{3}}=2|x|$. If the solar mass difference is generated by allowing a small perturbation in the $(1,1)$ entry in $M_{1 \nu}$, one gets

$$
M_{\nu}=\left(\begin{array}{ccc}
\epsilon & 0 & 0 \\
0 & x & \omega x \\
0 & \omega x & \omega^{2} x
\end{array}\right)
$$

with $\left|\frac{\epsilon}{2 x}\right| \approx \sqrt{\frac{\Delta m_{\text {sol }}^{2}}{\Delta m_{\text {atm }}^{2}}}$. The above matrix is diagonalized by

$$
U_{\nu}=V_{\nu}^{b} P_{12}
$$

where $P_{12}$ is a permutation matrix in 1-2 plane. This $U_{\nu}$ together with $U_{l}$ that diagonalizes $T_{l}$ modify the pattern $M_{2}$ by interchanging its first and second columns. This 
leads to $\sin ^{2} \theta_{12}=0.34$ without perturbing the successful predictions of $\theta_{23}$ and $\theta_{13}$. In this example, the original massless neutrino associated with the eigenvector $\frac{1}{\sqrt{3}}(1,1,1)^{T}$ becomes $m_{\nu_{2}}$ after the perturbation and the massless state arise due to the assumption $c= \pm \omega x$. A slight departure from this assumption leads to all massive neutrinos but without changing the mixing pattern as long as $|c \pm \omega x|<|\epsilon|$. Perturbation $\epsilon$ is small compared to the dominant mass $2|x|$ but needs to be assumed larger than the second mass. The perturbation matrix in the above example, i.e. $M_{2 \nu}=M_{\nu}-M_{1 \nu}=$ Diag. $(\epsilon, 0,0)$ satisfies $S_{2}^{T} M_{2 \nu} S_{2}=M_{2 \nu}$ with $S_{2}=$ Diag. $(1, \omega, \omega)$. The $S_{2}$ belongs to the group $\llbracket 162,10 \rrbracket$ and it is a product of two $S(3,0,1,0)$ which is one of the generators of this group. Hence it is

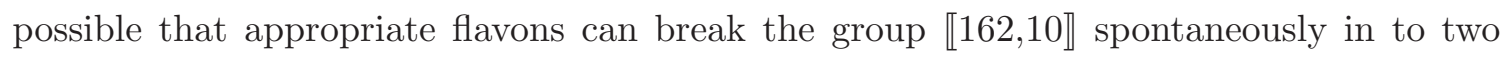
different subgroups characterized by $S_{1}$ and $S_{2}$ and generates two contributions $M_{1 \nu}$ and $M_{2 \nu}$ invariant under them, respectively. The full neutrino mass matrix $M_{\nu}=M_{1 \nu}+M_{2 \nu}$ is not invariant under $S_{1,2}$.

\subsubsection{The group $S_{4}(2)$}

The $S_{4}(2)$ is an order 48 group generated from specific matrices $S(4,1,3,1)$ and $T(4,3,3,1)$ given in table 1 . It is isomorphic to $A_{4} \rtimes Z_{4}$ and contains $S_{4}$ as a subgroup which has been discussed as possible symmetry for the TBM mixing [1-4]. Let us choose the following residual symmetries which are contained in $S_{4}(2)$ :

$$
S_{1}=\left(\begin{array}{ccc}
i & 0 & 0 \\
0 & 0 & -i \\
0 & i & 0
\end{array}\right), \quad S_{2}=\left(\begin{array}{ccc}
0 & 0 & -i \\
0 & -i & 0 \\
i & 0 & 0
\end{array}\right), \quad T_{l}=\left(\begin{array}{lll}
0 & 1 & 0 \\
0 & 0 & 1 \\
1 & 0 & 0
\end{array}\right)
$$

The pieces $M_{i \nu}$ of the neutrino mass matrix invariant under $S_{i}$ are given by:

$$
M_{1 \nu}=\left(\begin{array}{ccc}
0 & 0 & 0 \\
0 & x_{1} & a_{1} \\
0 & a_{1} & -x_{1}
\end{array}\right), \quad M_{2 \nu}=\left(\begin{array}{ccc}
x_{2} & 0 & a_{2} \\
0 & 0 & 0 \\
a_{2} & 0 & -x_{2}
\end{array}\right)
$$

The above structures of $M_{i \nu}$ may arise in specific models like the one recently proposed by us in [20]. Assuming that the three generations of lepton doublets $L_{L}$ transform as a triplet $3_{3}$ and a set of flavon fields $\phi_{i}^{\nu}, \chi_{i}^{\nu}(i=1,2)$ transform as $3_{2}$ and $2_{1}$ respectively under $S_{4}(2)$, one can write a leading order operator for the light Majorana neutrino masses $\operatorname{as}^{4}$

$$
\mathcal{W}_{\nu}=\sum_{i=1,2}\left(x_{i}^{\prime} L_{L} L_{L} \chi_{i}^{\nu}+a_{i}^{\prime} L_{L} L_{L} \phi_{i}^{\nu}\right) \frac{H_{u} H_{u}}{\Lambda^{2}},
$$

where $H_{u}$ is an $S_{4}(2)$ singlet Higgs doublet in the minimal supersymmetric standard model. The vacuum configuration $\left\langle\phi_{1}^{\nu}\right\rangle=v_{\phi_{1}}(1,0,0)^{T},\left\langle\phi_{2}^{\nu}\right\rangle=v_{\phi_{2}}(0,1,0)^{T},\left\langle\chi_{1}^{\nu}\right\rangle=v_{\chi_{1}}(1,0)^{T}$ and $\left\langle\chi_{2}^{\nu}\right\rangle=v_{\chi_{2}}(1, \sqrt{3})^{T}$ then leads to the forms of $M_{i \nu}$ given in eq. (5.10) with $x_{i}=$

\footnotetext{
${ }^{4}$ We refer reader to $[20,21]$ for the details of $S_{4}(2)$ group, its representations and the relevant tensor product decomposition rules.
} 
$x_{i}^{\prime} v_{\chi_{i}}\left\langle H_{u}\right\rangle^{2} / \Lambda^{2}$ and $a_{i}=a_{i}^{\prime} v_{\phi_{i}}\left\langle H_{u}\right\rangle^{2} / \Lambda^{2}$. Similarly, $M_{l} M_{l}^{\dagger}$ with the above residual symmetry can be obtained from the charged leptons Yukawa interactions similar to the one given in [20] but with a different vacuum structure $\left\langle\phi_{1}^{l}\right\rangle=v_{\phi_{1}^{l}}(1,1,1)^{T},\left\langle\phi_{2}^{l}\right\rangle=v_{\phi_{2}^{l}}\left(1, \omega, \omega^{2}\right)^{T}$ and $\left\langle\phi_{3}^{l}\right\rangle=v_{\phi_{3}^{l}}\left(1, \omega^{2}, \omega\right)^{T}$. Note that for a given $i=1$ or 2 , the vacuums of $\phi_{i}^{\nu}$ and $\chi_{i}^{\nu}$ leave the symmetry in $M_{i \nu}$ corresponding to $S_{i \nu}$ unbroken and each piece satisfies eq. (5.5). The combined matrix $M_{\nu}$ is not invariant under either but it still leads to interesting predictions. This follows from the fact that the neutrino mass matrix is controlled by four (instead of six) parameters $\left(x_{1,2}, a_{1,2}\right)$ and the charged lepton mixing matrix $U_{l}$ given by eq. (3.2) does not contain any free parameters. We shall assume $x_{1,2}, a_{1,2}$ to be real. In this case, the predictions of $M_{\nu}$ become transparent in the flavour basis with diagonal $M_{l} M_{l}^{\dagger}$ :

$$
M_{\nu f} \equiv U_{l}^{T} M_{\nu} U_{l}=U_{\omega}^{T} M_{\nu} U_{\omega}=\left(\begin{array}{ccc}
2 x & a & a^{*} \\
a & y & -x \\
a^{*} & -x & y^{*}
\end{array}\right)
$$

where $x, y, a$ are given in terms of $x_{1,2}, a_{1,2} . x$ is real and is related to four parameters in $y, a$ by:

$$
x=\frac{1}{3}(\operatorname{Re}(y-a)+\sqrt{3} \operatorname{Im}(y+a)) .
$$

$M_{\nu f}$ is invariant under the generalized $\mu-\tau$ symmetry [33-36] which combines CP and the $\mu-\tau$ symmetry. This symmetry appears here as an accidental symmetry of $M_{\nu f}$ when $x_{1,2}, a_{1,2}$ are real. It is known [33-36] that this symmetry leads to equality $\left|U_{\mu a}\right|=\left|U_{\tau a}\right|$ for $a=1,2,3$ and results in two predictions:

$$
\sin \theta_{13} \cos \delta=0, \quad \theta_{23}=\frac{\pi}{4}
$$

Other interesting prediction also emerges in the limiting case of real $a, y$. In this limit, $M_{\nu f}$ obeys $\mu-\tau$ symmetry and leads to $\theta_{13}=0, \theta_{23}=\frac{\pi}{4}$. Moreover, eq. (5.13) then implies that sum of elements in each of the rows of $M_{\nu f}$ is equal. As discussed at several places [1-4], this implies a $Z_{2}$ magic symmetry and $M_{\nu f}$ in this limit is thus invariant under a $Z_{2} \times Z_{2}$ symmetry which implies TBM mixing. Introducing imaginary part in $a, y$ can lead to the required deviation from the TBM mixing. The following choice,

$$
\left(a_{1}, a_{2}, x_{1}, x_{2}\right)=(0.02369,0.00512,0.03159,-0.05003) \mathrm{eV}
$$

minimizes the relevant $\chi^{2}$ and leads to the central values for $\theta_{12}, \theta_{13}$ and the solar to atmospheric mass ratio. Overall scale is fixed from the value of the atmospheric mass scale. The resulting masses are

$$
\left(m_{\nu_{1}}, m_{\nu_{2}}, m_{\nu_{3}}\right)=(0.0497,0.0505,0.0008) \mathrm{eV} .
$$

This pattern corresponds to the inverted hierarchy. The atmospheric mixing angle and CP phase remain maximal as they follow the relations, eq. (5.14) which are independent of the choice of parameters. The present data however favor non-maximal $\theta_{23}$ in the first 
quadrant. This can be accommodated here by allowing complex $x_{1,2}, a_{1,2}$. A possible but not unique choice giving correct central values for all the mixing angles and $\Delta m_{\mathrm{sol}}^{2}, \Delta m_{\mathrm{atm}}^{2}$ is given by:

$$
\left(a_{1}, a_{2}, x_{1}, x_{2}\right)=(-0.01327+0.06426 i,-0.00022,-0.04039,0.08653) \mathrm{eV}
$$

Before closing, let us note some interesting aspects of two contributions in $M_{\nu} . M_{i \nu} M_{i \nu}^{\dagger}$ is diagonal and contains one massless and two degenerate states for each $i=1,2$ when $x_{1,2}, a_{1,2}$ are real. Thus if only one of the contributions is present in $M_{\nu}$ then the neutrino mixing matrix is given by $U_{\omega}^{\dagger} R$, where $R$ denotes a rotation in the space of the degenerate eigenvalue by an arbitrary angle. The combined effect of two contributions is to lift degeneracy and generate the solar scale and to give the correct mixing angles. The massless state implied by each contribution is trimaximally mixed. The combined matrix has a state with negligible mass and is almost bimaximally mixed, see eqs. (5.15), (5.16).

\section{Summary}

We explored in detail our recent proposal [20] of predicting a massless Majorana neutrino and lepton mixing angles at the leading order by assuming that the neutrino and the charged lepton symmetries appropriate for this are contained in some flavour group $G_{f}$. As argued, the DSG of U(3) are sufficient to provide at least one massless neutrino. Using available information on such groups, we carried out detailed analysis for their viability to predict a massless neutrino and suitable lepton mixing patterns at the leading order. The study encompassed very large number of groups which include all DSG in class (X) defined earlier. As we discussed, their viability as flavour symmetry and resulting mixing pattern can be worked out analytically. Main findings applicable to groups in class (X) are:

- For all the groups in class (X), a massless neutrino can remain unmixed or it can be either trimaximally or bimaximally mixed. This result holds irrespective of the order of the group and is applicable to several known infinite group series. The above structures of the massless state leads to incorrect solar mixing angle at the leading order.

- The groups in this category can however lead to a good zeroth order prediction namely, bimaximal for the mixing matrix in case of the inverted hierarchy which always predicts this pattern as shown analytically.

- In case of the normal hierarchy, the different values of $\theta_{23}$ and $\theta_{13}$ can be predicted depending on the group under consideration. The relation $\sin ^{2} \theta_{12} \approx \frac{2}{3} \cos ^{2} \theta_{13}$ persists as explained above and the Dirac CP phase is trivial in all such cases.

We further numerically investigated 75 groups of order $<512$ tabulated by Ludl [21]. 70 of these fall in category $(\mathrm{X})$ and only the six of these are argued to qualify to be 
a symmetry of the lepton sector. The mixing patterns implied by these groups for the normal hierarchy are listed in table 2. All six can also lead to BM mixing with an inverted hierarchy.

The remaining 5 groups are also analyzed numerically and only one of them, namely

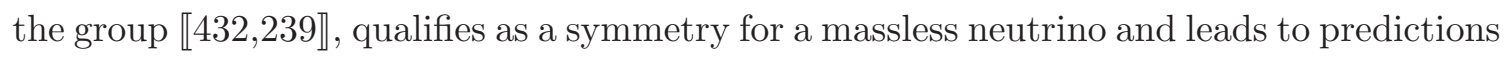
for all three mixing angles fairly close to their best fit values in case of normal hierarchy. This group thus provides a sole example of a flavour symmetry with massless neutrino and correct predictions for all the mixing angles. A solution with inverted hierarchy is also found in this case with the BM values of $\theta_{13}$ and $\theta_{23}$ but with $\sin ^{2} \theta_{12}=0.21$.

Alternative ways to modify or avoid the unsatisfactory prediction of the solar angle are also discussed briefly. These include relaxing assumption of leptons transforming as a 3dimensional irreducible representation and allowing more complicated residual symmetries. One can avoid wrong prediction for the solar angle in the former case but it remains unpredicted. In this case, an inverted hierarchy solution with $\mu-\tau$ symmetry pattern can be obtained from the groups like $\Sigma\left(2 N^{2}\right)$ with $N \geq 3$ or even from their subgroups if $N$ is sufficiently large. In the latter case, one can obtain all the mixing angles correctly as shown through two examples based on the group $\llbracket 162,10 \rrbracket$ and $S_{4}(2)$.

\section{Acknowledgments}

A.S.J. thanks the Department of Science and Technology, Government of India for support under the J. C. Bose National Fellowship programme, grant no. SR/S2/JCB-31/2010. K.M.P. thanks the Department of Physics and Astronomy of the University of Padova for its support. He also acknowledges partial support from the European Union network FP7 ITN INVISIBLES (Marie Curie Actions, PITN-GA-2011-289442).

Open Access. This article is distributed under the terms of the Creative Commons Attribution License (CC-BY 4.0), which permits any use, distribution and reproduction in any medium, provided the original author(s) and source are credited.

\section{References}

[1] G. Altarelli and F. Feruglio, Discrete flavor symmetries and models of neutrino mixing, Rev. Mod. Phys. 82 (2010) 2701 [arXiv:1002.0211] [INSPIRE].

[2] G. Altarelli, F. Feruglio and L. Merlo, Tri-bimaximal neutrino mixing and discrete flavour symmetries, Fortsch. Phys. 61 (2013) 507 [arXiv:1205.5133] [INSPIRE].

[3] A.Y. Smirnov, Discrete symmetries and models of flavor mixing, J. Phys. Conf. Ser. 335 (2011) 012006 [arXiv:1103.3461] [InSPIRE].

[4] S.F. King and C. Luhn, Neutrino mass and mixing with discrete symmetry, Rept. Prog. Phys. 76 (2013) 056201 [arXiv:1301.1340] [INSPIRE].

[5] H. Ishimori et al., Non-abelian discrete symmetries in particle physics, Prog. Theor. Phys. Suppl. 183 (2010) 1 [arXiv:1003.3552] [INSPIRE]. 
[6] C.S. Lam, Symmetry of lepton mixing, Phys. Lett. B 656 (2007) 193 [arXiv:0708.3665] [INSPIRE].

[7] C.S. Lam, Determining horizontal symmetry from neutrino mixing, Phys. Rev. Lett. 101 (2008) 121602 [arXiv:0804.2622] [INSPIRE].

[8] C.S. Lam, The unique horizontal symmetry of leptons, Phys. Rev. D 78 (2008) 073015 [arXiv:0809.1185] [INSPIRE].

[9] C.S. Lam, Finite symmetry of leptonic mass matrices, Phys. Rev. D 87 (2013) 013001 [arXiv: 1208.5527] [INSPIRE].

[10] C.S. Lam, A bottom-up analysis of horizontal symmetry, arXiv:0907.2206 [INSPIRE].

[11] R.d.A. Toorop, F. Feruglio and C. Hagedorn, Discrete flavour symmetries in light of T2K, Phys. Lett. B 703 (2011) 447 [arXiv:1107.3486] [INSPIRE].

[12] R. de Adelhart Toorop, F. Feruglio and C. Hagedorn, Finite modular groups and lepton mixing, Nucl. Phys. B 858 (2012) 437 [arXiv:1112.1340] [INSPIRE].

[13] D. Hernandez and A.Y. Smirnov, Lepton mixing and discrete symmetries, Phys. Rev. D 86 (2012) 053014 [arXiv:1204.0445] [INSPIRE].

[14] D. Hernandez and A.Y. Smirnov, Discrete symmetries and model-independent patterns of lepton mixing, Phys. Rev. D 87 (2013) 053005 [arXiv: 1212.2149] [INSPIRE].

[15] I. de Medeiros Varzielas and L. Lavoura, Golden ratio lepton mixing and nonzero reactor angle with $A_{5}$, J. Phys. G 41 (2014) 055005 [arXiv: 1312.0215] [INSPIRE].

[16] L. Lavoura and P.O. Ludl, Residual $Z_{2} \times Z_{2}$ symmetries and lepton mixing, arXiv: 1401.5036 [INSPIRE].

[17] C.S. Lam, Group theory and dynamics of neutrino mixing, Phys. Rev. D 83 (2011) 113002 [arXiv:1104.0055] [INSPIRE].

[18] M. Holthausen, K.S. Lim and M. Lindner, Lepton mixing patterns from a scan of finite discrete groups, Phys. Lett. B 721 (2013) 61 [arXiv:1212.2411] [INSPIRE].

[19] D. Hernandez and A.Y. Smirnov, Relating neutrino masses and mixings by discrete symmetries, Phys. Rev. D 88 (2013) 093007 [arXiv:1304.7738] [INSPIRE].

[20] A.S. Joshipura and K.M. Patel, Horizontal symmetries of leptons with a massless neutrino, Phys. Lett. B 727 (2013) 480 [arXiv:1306.1890] [INSPIRE].

[21] P.O. Ludl, On the finite subgroups of $U(3)$ of order smaller than 512, J. Phys. A 43 (2010) 395204 [Erratum ibid. A 44 (2011) 139501] [arXiv:1006.1479] [INSPIRE].

[22] K.M. Parattu and A. Wingerter, Tribimaximal mixing from small groups, Phys. Rev. D 84 (2011) 013011 [arXiv: 1012.2842] [INSPIRE].

[23] P.O. Ludl, Systematic analysis of finite family symmetry groups and their application to the lepton sector, arXiv:0907.5587 [INSPIRE].

[24] W. Grimus and P.O. Ludl, Finite flavour groups of fermions, J. Phys. A 45 (2012) 233001 [arXiv:1110.6376] [INSPIRE]. 
[25] W. Grimus and P. Ludl, Principal series of finite subgroups of SU(3), J. Phys. A 43 (2010) 445209 [arXiv:1006.0098] [inSPIRE].

[26] A. Merle and R. Zwicky, Explicit and spontaneous breaking of SU(3) into its finite subgroups, JHEP 02 (2012) 128 [arXiv:1110.4891] [INSPIRE].

[27] W. Grimus and P.O. Ludl, On the characterization of the $\mathrm{SU}(3)$-subgroups of type $C$ and $D$, arXiv:1310.3746 [INSPIRE].

[28] The GAP Group, GAP - Groups, Algorithms, and Programming, Version 4.7.2, www.gap-system.org.

[29] F. Capozzi, G. Fogli, E. Lisi, A. Marrone, D. Montanino et al., Status of three-neutrino oscillation parameters, circa 2013, arXiv:1312.2878 [INSPIRE].

[30] M. Holthausen and K.S. Lim, Quark and leptonic mixing patterns from the breakdown of a common discrete flavor symmetry, Phys. Rev. D 88 (2013) 033018 [arXiv:1306.4356] [INSPIRE].

[31] C. Hagedorn, A. Meroni and L. Vitale, Mixing patterns from the groups $\Sigma(n \phi)$, J. Phys. A 47 (2014) 055201 [arXiv:1307.5308] [inSPIRE].

[32] S. Gupta, A.S. Joshipura and K.M. Patel, How good is $\mu-\tau$ symmetry after results on non-zero $\theta_{13}$ ?, JHEP 09 (2013) 035 [arXiv: 1301.7130] [INSPIRE].

[33] P.F. Harrison and W.G. Scott, $\mu-\tau$ reflection symmetry in lepton mixing and neutrino oscillations, Phys. Lett. B 547 (2002) 219 [hep-ph/0210197] [INSPIRE].

[34] K. Babu, E. Ma and J. Valle, Underlying $A_{4}$ symmetry for the neutrino mass matrix and the quark mixing matrix, Phys. Lett. B 552 (2003) 207 [hep-ph/0206292] [INSPIRE].

[35] W. Grimus and L. Lavoura, A nonstandard CP transformation leading to maximal atmospheric neutrino mixing, Phys. Lett. B 579 (2004) 113 [hep-ph/0305309] [INSPIRE].

[36] S. Gupta, A.S. Joshipura and K.M. Patel, Minimal extension of tri-bimaximal mixing and generalized $Z_{2} \times Z_{2}$ symmetries, Phys. Rev. D 85 (2012) 031903 [arXiv:1112.6113] [INSPIRE]. 